\title{
Discriminação Auditiva-Visual em Deficientes Auditivos Pós-Linguais com Implante Coclear
}

\author{
Fabiane da Silva Pereira ${ }^{1}$ \\ Programa de Pós-Graduação em Teoria e Pesquisa do Comportamento, Universidade \\ Federal do Pará, Belém, PA, Brasil \\ Suelen Nicole da Silva Lobato \\ Programa de Pós-Graduação em Neurociências e Comportamento, Universidade \\ Federal do Pará, Belém, PA, Brasil \\ Juliana Sequeira César de Oliveira \\ Universidade Federal do Pará, Belém, PA, Brazil \\ Cyntia Tzue Yamaguchi \\ José Claudio de Barros Cordeiro \\ Hospital Universitário Bettina Ferro de Souza, Universidade Federal do Pará, \\ Belém, PA, Brasil \\ Olavo de Faria Galvão \\ Programa de Pós-Graduação em Neurociências e Comportamento, Universidade \\ Federal do Pará, Belém, PA, Brasil
}

\section{Resumo}

Com o objetivo de verificar o efeito do treino do comportamento de ouvinte sobre o de falante, e especificamente verificar se deficientes auditivos pós-linguais usuários de implante coclear: (a) aprenderiam discriminações condicionais auditivo-visuais com estímulos convencionais e não convencionais; (b) formariam classes dos estímulos correlacionados; (c) apresentariam desempenho generalizado para outras frequências auditivas; e (d) se estes repertórios afetariam o comportamento de falante, dois adultos com deficiência auditiva pós-lingual usuários de implante coclear foram expostos a um programa de ensino com questões de escolha de acordo com o modelo, em sete fases: 1) Pré-treino de tarefas auditivo-visuais com utilização do fading out; 2) Pré-teste de nomeação para avaliar o comportamento de falante; 3) Ensino das relações entre palavras ditadas e figuras; 4) Ensino das relações entre palavras ditadas e escritas; 5) Teste de formação de classes; 6) Teste de generalização auditiva e 7) Pós-teste de nomeação. Os participantes aprenderam as relações ensinadas, formaram classes dos estímulos correlacionados e generalizaram o desempenho para uma voz de timbre diferente. Comparando-se os pré e pós-testes, observou-se aumento no índice de correspondência do comportamento de falante com a comunidade verbal. Discutem-se as possibilidades dessa metodologia para a reabilitação da fala nessa população.

Palavras-chave: Discriminação auditiva-visual, equivalência de estímulos, implante coclear.

Endereço para correspondência: Conjunto Providência, Quadra 22, Rua 7, no 411, Val-de-Cans, Belém, PA, Brasil 66110-101. Fones: 91 8110-1489/3257-9505. E-mail: fabpsi@gmail.com

Os dados relatados neste artigo foram extraídos da pesquisa de mestrado "Discriminação auditivo-visual em adultos com deficiência auditiva pós-lingual e implante coclear" da primeira autora sob orientação do quinto autor. Financiamentos: Bolsa de Mestrado Coordenação de Aperfeiçoamento de Pessoal de Nível Superior (CAPES) à primeira autora; Bolsa de Produtividade em Pesquisa P313514/2014-3 ao quinto autor; Instituto Nacional de Ciência e Tecnologia Sobre Comportamento, Cognição e Ensino. Conselho Nacional de Desenvolvimento Científico e Tecnológico - CNPQ (Processo \#573972/2008-7) e Fundação de Amparo à Pesquisa do Estado de São Paulo - FAPESP (Processo \#2008/57705-8). 


\title{
Auditory-Visual Discrimination in Adults with Postlingual Hearing Impairment and Cochlear Implants
}

\begin{abstract}
Aiming the evaluation of listener-behavior training on speaker-behavior, specifically if individuals with hearing impairment and cochlear implant would: (a) learn audio-visual conditional discriminations with conventional and non-conventional words; (b) form stimulus classes; (c) present generalized responses to other audio frequencies; and (d) transfer the acquired hearing competences to speaker repertoires, two adults with post-lingual hearing loss and cochlear implant were exposed to a seven phases' matching to sample conditional discriminations teaching program consisting of: 1) Pre-training of auditory-visual tasks using fading out; 2) Pre-test of naming to evaluate the speaker behavior, 3) Teaching relations between dictated words and figures; 4) Teaching relations between dictated and written words, 5) Test of class formation; 6) Test of hearing generalization; 7) Post-test of naming. The participants learned the directly taught relations, demonstrated class formation and generalization. Comparing pre- and post-tests, they showed higher correspondence between their speaker behavior and verbal community's speaker behavior. The possibilities of this methodology for speaking rehabilitation in this population is discussed.
\end{abstract}

Keywords: Auditory-visual discrimination, stimulus equivalence, cochlear implant.

\section{Discriminación Visual-Auditiva en Adultos con Discapacidad Auditiva Postlocutiva y Implante Coclear}

\section{Resumen}

Con el objetivo de averiguar el efecto del entrenamiento de la conducta de oyente sobre la de hablante, verificar específicamente si discapacitados auditivos post-locutivos usuarios de IC: (a) aprenderían la discriminación condicional auditivo-visual de estímulos convencionales y no convencionales; (b) formarían clases de estímulos; (c) generalizarían a otra frecuencia; (d) si estos repertorios influyen en el comportamiento del orador, dos adultos con discapacidad auditiva post-lingual y usuarios de implante coclear fueron expuestos a un programa de enseñanza con cuestiones de selección por igualación a la muestra, en siete fases: 1) Pre-entrenamiento de tareas visual-auditivas con utilización del desvanecimiento (fading out); 2) Prueba previa de nombramiento para evaluar la conducta de hablante; 3) Entrenamiento de relaciones entre palabras dictadas y figuras; 4) Entrenamiento de relaciones entre palabras dictadas y escritas; 5) Prueba de formación de clases; 6) Prueba de generalización auditiva y 7) Prueba posterior de nombramiento. Los participantes aprendieron las relaciones enseñadas, demostraron formación de clases y generalización. La comparación de los pre y post tests mostró que, ellos presentaran índices de correspondencia más grandes con la comunidad verbal en el comportamiento de hablar. Se discute la importancia de esta metodología para la rehabilitación en esta población.

Palabras clave: Discriminación visual-auditiva, equivalencia de estímulos, implante coclear.

O paradigma da equivalência de estímulos tem sido usado como tecnologia comportamental para a reabilitação auditiva de indivíduos com surdez pré e pós-lingual, cuja sensibilidade auditiva foi restaurada via implante coclear (IC), independentemente do tempo de privação.
O IC é um dispositivo eletrônico com terminais inseridos cirurgicamente no ouvido interno, mais especificamente no órgão de Corti, que transforma a energia sonora em pulsos elétricos conduzidos pelo nervo auditivo no qual o processamento neural da audição se inicia. É indi- 
cado para indivíduos com deficiência auditiva neurosensorial, bilateral, com grau de profundo a severo, com tempo de aquisição da surdez tanto pré quanto pós-lingual (Bevilacqua, 1998; Clark, 1996).

Em um modelo simplificado dos comportamentos de ouvinte e de falante via ensino de relações condicionais entre figuras e palavras impressas com estímulos auditivos, o paradigma da equivalência de estímulos tem sido usado no estudo de usuários de IC para estabelecer relações entre ver, ouvir e falar. O procedimento inclui teste da emergência de relações não ensinadas diretamente entre esses estímulos visuais e a nomeação oral dos mesmos.

$\mathrm{O}$ uso do paradigma de equivalência para a investigação da funcionalidade do implante coclear ainda é recente. Trabalhos com crianças com deficiência auditiva pré e pós-lingual, utilizaram a noção de currículo comportamental para instalar repertórios de ouvinte, iniciando pelo ensino de tarefas mais simples até as mais complexas seja com estimulação diretamente na cóclea (da Silva et al., 2006), com estímulos linguísticos convencionados e não convencionados (pseudopalavras; Almeida-Verdu et al., 2008), ou com avanço dos repertórios mais complexos como o ensino de leitura de sentenças (Golfeto $\&$ de Souza, 2015).

Neves (2014) revisou as pesquisas que avaliavam efeitos do procedimento de ensino sobre os comportamentos de falante e ouvinte de pessoas com IC e observou que a maioria dos estudos foi conduzido com crianças com deficiência auditiva pré-lingual. Na sequência, Neves, Verdu, MortariMoret e Silva (2015) analisaram o estado da arte das pesquisas envolvendo linguagem e IC e evidenciou um aumento de publicações com a temática, principalmente com objetivos de avaliação e identificação de possíveis fatores que interferem nos processos de linguagem em usuários de IC. De todas as publicações analisadas, entretanto, apenas duas em 86 realizaram estudos com participantes adultos usuários de IC pós-linguais. Este estudo, portanto, busca diminuir a escassez de pesquisas com a população adulta usuária de IC usando o modelo de equivalência de estímulos como recurso metodológico. Este estudo é também pioneiro na região de onde provêm os seus participantes, onde, à época do estudo, a maior parte das pessoas submetidas à cirurgia de IC era de adultos com deficiência auditiva pós-lingual.

Os dados iniciais demonstraram variedade de desempenho das crianças, principalmente aquelas com um tempo maior de privação auditiva e menor tempo de implante que as demais. $\mathrm{Na}$ discussão os autores relacionam o desempenho abaixo do esperado à falta de dicas orofaciais nas tarefas apresentadas via computador (Almeida-Verdu, de Souza, Bevilacqua, \& Souza, 2009; Souza, Almeida-Verdu, \& Bevilacqua, 2013).

Trabalhos mais recentes investigaram o procedimento de exclusão como forma de levar estímulos visuais e auditivos a pertencer a uma mesma classe, facilitando a emergência de nomeação de figuras e leitura de palavras (Anastácio-Pessan, Almeida-Verdu, Bevilacqua, \& Souza, 2015; Golfeto \& de Souza, 2015).

O procedimento de ensino por exclusão consiste no aproveitamento de uma característica comportamental, presente também em crianças pequenas, de, diante de um problema de escolha entre duas alternativas para comparação, tender a escolher a alternativa nova para resolver o problema que se configura como novo, quando a outra alternativa já havia sido correta para outro problema. $\mathrm{O}$ uso desse procedimento tem sido promissor, visto que com ele a aprendizagem das relações entre palavras ditadas, palavras escritas e figuras requer menos tarefas (McIlvane, Munson, \& Stoddard, 1988).

Os resultados dessas pesquisas têm demonstrado que, de maneira geral, a aprendizagem de discriminações auditivas-visuais é gradativa, e que o comportamento de ouvinte é mais rapidamente apresentado que o comportamento de falante. Todos os estudos têm demonstrado formação de classes com palavras convencionadas e pseudopalavras ao longo de sucessivos testes (Anastácio-Pessan et al., 2015) além da aprendizagem de leitura de sentenças e fala inteligível (Golfeto \& de Souza, 2015).

Os implantados pós-linguais aprenderam, durante o período da surdez, a utilizar-se de meios alternativos à audição para compreender 
e interagir, como, por exemplo, a leitura labial, dificultando a aquisição de função dos estímulos auditivos. O contexto, a expressão facial e os movimentos labiais dos interlocutores facilitam a compreensão quando os estímulos auditivos são insuficientes. Para implantados com esse perfil, o ensino de relações entre palavras e pseudopalavras ditadas e figuras correspondentes em condições controladas com o uso do paradigma da equivalência de estímulos, foi adotado como uma forma de garantir que o responder se baseie apenas nos estímulos auditivos, evitando, assim, meios auxiliares acima mencionados continuem presentes e interferindo. $\mathrm{O}$ uso de pseudopalavras estabelece uma condição de controle para investigar a aprendizagem de relações em que dicas adicionais não estão disponíveis mesmo em deficientes auditivos pós-linguais com código oral estabelecido.

Considerando-se a relevância da adaptação do paradigma para dispor aos implantados essa tecnologia de apoio à construção do repertório de comunicação linguística oral, os objetivos do estudo foram avaliar as variáveis de procedimento envolvidas no treino de discriminações auditivo-visuais com relações condicionais entre palavras e pseudopalavras ditadas, figuras e palavras escritas e verificar se o treino facilitaria a emergência de vocalizações com correspondência total, e generalização para outras frequências de sons, em deficientes auditivos pós-linguais usuários de IC. Em síntese, verificar se deficientes auditivos pós-linguais usuários de IC: (a) aprenderiam discriminações condicionais auditivo-visuais com estímulos convencionais e não convencionais; (b) se formariam classes de estímulos; (c) se generalizariam para outras frequências auditivas; e (d) se estes repertórios afetariam o comportamento de falante.

\section{Método}

\section{Participantes}

Participaram do estudo um homem e uma mulher, Claudio e Ana (nomes fictícios), com deficiência auditiva neurossensorial profunda, bilateral, pós-lingual, usuários de implante coclear. Ambos com 45 anos e ensino fundamen- tal. No início do estudo, Cláudio apresentava cinco meses de privação auditiva e quatro meses de uso do IC (modelo Nucleus 24) e um ano e dois meses ao fim do estudo. Ana apresentava nove anos de privação auditiva e oito meses de uso do IC (modelo Med El) no início do estudo e um ano e três meses ao fim do estudo.

\section{Procedimentos Éticos}

Os participantes assinaram o Termo de Consentimento Livre e Esclarecido da pesquisa aprovada pelo Comitê de Ética em Pesquisa com Seres Humanos do Instituto de Ciências da Saúde da Universidade Federal do Pará (CAEE 0268. 0. 073. 000-12 e parecer 039/12).

\section{Instrumentos}

Foi utilizado um computador com tela sensível ao toque e o software EAM V. 4.0.04, desenvolvido por Dráuzio Capobianco e modificado por Carlos Picanço (Souza, 2010).

Foi utilizado como sistema de áudio um monitor de referência utilizado em estúdios profissionais, que possui alta fidelidade na reprodução de sons, com dimensão do gabinete de $15,0 \times 8,5 \times 10,0 \mathrm{~cm}$, com blindagem magnética. A resposta de frequência era de $45 \mathrm{~Hz}$ a $18 \mathrm{~Hz}$, com sensibilidade de $88 \mathrm{~dB}$, e frequência de crossover de $2,5 \mathrm{kHz}$.

Foram utilizados estímulos auditivos e visuais. Como estímulos auditivos foram apresentados sons gravados em vozes feminina e masculina adultas, e como estímulos visuais figuras e palavras escritas apresentadas em "janelas" quadradas com cerca de cinco centímetros de lado, em nove possíveis posições de uma matriz $3 \mathrm{x}$ 3 , na tela do monitor. Os estímulos apresentados foram de natureza convencionada e não-convencionada (pseudopalavras com constituição fonológica possível em nossa comunidade verbal, contudo sem significado agregado). Foram utilizadas palavras mono, di, tri e polissilábicas e figuras correspondentes. As palavras não-convencionadas eram dissilábicas, retiradas do estudo de Anastácio-Pessan et al. (2015) e as figuras retiradas da internet (estímulos não-convencionados). As figuras utilizadas no treino podem ser vistas na Figura 1. 


\begin{tabular}{|c|c|c|c|c|c|c|c|}
\hline \multicolumn{4}{|c|}{ Estímulos de linha de base } & \multicolumn{4}{|c|}{ Estímulos não convencionais } \\
\hline Auditivos & Figuras & & Palavras escritas & Auditivos & Figuras & & Palavras escritas \\
\hline /Mão/ (A1) & & (B1) & MÃO (C1) & /Pafe/(A5) & & (B5) & PAFE (C5) \\
\hline /kaho/(A2) & & ( (B2) & CARRO (C2) & /Duka/(A6) & & (B6) & DUCA (C6) \\
\hline /kavalo/ (A3) & & (B3) & CAVALO (C3) & /Tiba/ (A7) & & (B7) & TIBA (C7) \\
\hline /abakafi/(A4) & & (B4) & ABACAXI (C4) & /Zigo/ (A8) & & (B8) & ZIGO (C8) \\
\hline
\end{tabular}

Figura 1. Estímulos utilizados nas Fases 3 a 6 deste estudo.

\section{Procedimento de Coleta de Dados}

O programa de ensino pretendeu fortalecer as habilidades auditivas dos participantes expondo-os a tarefas nas quais deveriam atentar aos estímulos sonoros e escolher a figura ou palavra escrita correspondente. Posteriormente, foram expostos a testes de relações derivadas das ensinadas, e ainda foram testadas as habilidades de falante em tarefas de nomeação e leitura.

$\mathrm{O}$ ambiente experimental consistia em uma sala iluminada e climatizada artificialmente, com um computador sensível ao toque, mouse, sistema de som e uma cadeira. O participante ficava sentado em frente ao monitor, no qual eram apresentadas tentativas de emparelhamento ao modelo, que começavam com a apresentação de um estímulo modelo visual ou auditivo-visual; ao tocar no estímulo este desaparecia e eram apresentados quatro estímulos de comparação, um dos quais, definido como correto, que se tocado produzia uma consequência para acerto, com a frase "Você acertou" apresentada na tela imediatamente após a resposta do participante e um intervalo de três segundos até que uma nova tentativa estivesse disponível.. Em caso de respostas incorretas, uma tela preta seguia a resposta por três segundos e uma nova tentativa era apresentada. Foram realizadas sete fases que envolveram tarefas de treino e de teste. A consequência programada para acerto era disponibilizada apenas nas tentativas de treino. As fases do procedimento estão resumidas na Tabela $1 \mathrm{e}$ descritas a seguir.

Fase 1. Os participantes foram expostos a um pré-teste, no qual receberam a instrução para nomear figuras e ler palavras com o objetivo de avaliar suas vocalizações e selecionar os estímulos convencionados que comporiam as fases seguintes. Não houve consequência programada para as respostas durante esta fase. Foram usadas 32 figuras e as 32 palavras correspondentes aos nomes dessas figuras. As figuras foram escolhidas com base nos seus nomes e com base na categoria silábica à qual pertenciam (mono, di, tri e polissilábicas) pelos nomes que apresentavam, sendo oito estímulos para cada categoria silábica. Com base no desempenho dos participantes, foram selecionados quatro estímulos convencionados vocalizados com correspondência total durante o teste de nomeação de figuras.

Fase 2. Com o objetivo de estabelecer controle pelo estímulo auditivo foi feito um pré-treino auditivo-visual com apresentação 
Tabela 1

Sequência de Tarefas das Fases de Ensino Empregadas neste Estudo, os Repertórios-Alvo em cada Fase, Estímulos e o Critério Utilizado para Passagem de Fase

\begin{tabular}{ccccc}
\hline Fase & Tarefa & Repertório alvo & Estímulos & Critério \\
\hline 1 & Pré-teste & $\begin{array}{c}\text { Avaliação de leitura e } \\
\text { nomeação de figuras }\end{array}$ & Convencionados & - \\
2 & Pré-treino & IDMTS e AVMTS & Convencionados & $90 \%$ \\
3 & Ensino AB & AVMTS & Convencionados e não convencionados & $90 \%$ \\
4 & Ensino AC & AVMTS & Convencionados e não convencionados & $90 \%$ \\
5 & Teste de formação de classe & CB/BC & Não convencionados & - \\
6 & Teste de generalização & AVMTS & Não convencionados & - \\
7 & Pós-teste & & Convencionais & - \\
\hline
\end{tabular}

Notas. Pareamento ao Modelo por Identidade (IDMTS), do inglês Identity Matching to Sample; Pareamento ao Modelo Auditivo-Visual (AVMTS), do inglês Auditive-Visual Matching to Sample.

de modelo composto, isto é, som e figura simultaneamente, com desaparecimento gradual (Fading out) da figura em uma transparência aumentada em três passos $(25 \%, 50 \%$ e $75 \%$ ), até permanecer um quadrado amarelo independentemente de qual era o som. Para a realização do pré-treino, foram utilizados quatro estímulos com nomes dissílabos: bola, fogo, sino e casa, as mesmas usadas por AnastácioPessan et al. (2015). Estes estímulos não foram reapresentados em outras fases do estudo. Apenas escolhas corretas eram reforçadas.

Nas Fases 3 e 4, apresentadas a seguir, utilizou-se o procedimento de discriminação auditivo-visual para o ensino por exclusão. Foram utilizados os estímulos convencionados selecionados no pré-teste (linha de base) e estímulos não convencionados.

Fase 3. Deu-se início ao treino das relações entre palavras ditadas (Conjunto A) e figuras (Conjunto $\mathrm{B}$ ) via procedimento de exclusão (Dixon, 1977; Mcllvane \& Stoddard, 1981), e inseridos quatro estímulos não-convencionados (ver Figura 1). O treino foi apresentado em uma sequência de quatro blocos de tentativas, nos quais as relações treinadas variaram, conforme descrito a seguir:

- Bloco 1 - Linha de base: discriminação condicional auditivo-visual com estímulos convencionados selecionados no pré-teste (linha de base)
- Bloco 2 - Verificação da aprendizagem por exclusão: discriminação condicional auditivo-visual com estímulos não-convencionadas. As tentativas eram compostas por um som não-convencionado com a função de estímulo-modelo, por exemplo, /pafe/, e de quatro figuras como estímulos-comparação, das quais três eram de linha de base e uma figura era não-convencionada correspondente ao modelo. Nesta condição, o participante poderia selecionar a figura não-convencionada por meio de exclusão das figuras conhecidas, ou estabelecer uma relação direta entre som e figura não-convencionados. Não houve reforçamento para as respostas corretas e erradas.

- Bloco 3 - Controle por novidade: o som apresentado como estímulo-modelo sempre era pertencente à linha de base e as figuras apresentadas como estímulos-comparação incluíam três figuras não-convencionadas e uma figura de linha de base. Nessa situação, o participante deveria escolher a figura correspondente ao som, rejeitando as figuras não-convencionadas. A escolha de uma relação de linha de base indicaria a manutenção desta e permitiria descartar um possível controle pela novidade que pudesse ter sido estabelecido nas tentativas do Bloco 2.

- Bloco 4 - Aprendizagem: neste bloco, foram apresentadas apenas relações não-con- 
vencionadas, isto é, tanto o som apresentado como estímulo-modelo quanto as figuras apresentadas como estímulos-comparação eram não-convencionados.

- Os blocos de tentativas eram apresentados sucessivamente, com 12 tentativas cada, independentemente do número de acertos. Caso o participante apresentasse $100 \%$ de acertos nos Blocos 1, 2 e 3 e não alcançasse este desempenho no Bloco 4, o que era esperado nas primeiras sessões, as sessões seguintes seriam compostas por 24 tentativas apenas das relações não-convencionadas, sendo seis tentativas para cada relação indefinida. $\mathrm{O}$ critério de aprendizagem era de $90 \%$ de acertos em todos os blocos de tentativas.

Fase 4. Treino das relações entre palavras ditadas (Conjunto A) e palavras escritas (Conjunto $\mathrm{C}$ ). $\mathrm{O}$ procedimento aplicado durante a Fase 4 teve as mesmas características da Fase 3, apenas com a substituição dos estímulos do conjunto B (figuras) para os estímulos do conjunto $\mathrm{C}$ (palavras escritas). O critério para passagem de fase, também, foi de $90 \%$ de acertos em todos os blocos de tentativas.

Fase 5. Teste das relações transitivas e simétricas. Após alcance de critério de aprendizagem, os participantes foram expostos a um teste $\mathrm{CB} / \mathrm{BC}$ de formação de classes, no qual avaliou-se as relações $\mathrm{CB}$, entre palavras escritas (Conjunto C) e figuras (Conjunto B) e BC, entre figuras como modelo (Conjunto B) e palavras escritas (Conjunto C) como estímulos para escolha. Foram apresentadas 40 tentativas, sendo 24 tentativas de teste e 16 tentativas de linha de base em quatro blocos de tentativas

Fase 6. Teste de generalização. Os participantes foram expostos a um novo teste, com características semelhantes à Fase 5, de generalização auditiva, para verificar se as relações se mantinham quando as palavras apresentadas estivessem gravadas com outra entonação, uma voz masculina. O objetivo deste teste foi verificar se após apresentar responder acurado diante de relações entre sons e figuras não convencionadas, os participantes poderiam responder, ainda com acurácia, a sons com frequências diferentes aos apresentados durante o treino. Foram apresentadas 32 tentativas, oito de linha de base e 24 de teste. Independentemente do número de acertos, o participante era exposto à fase seguinte

Fase 7. Pós-teste. Igual ao pré-teste (Fase 1), de nomeação de figuras e leitura de palavras, com o objetivo de avaliar o efeito do treino sobre as vocalizações.

\section{Procedimentos de Análise de Dados}

Avaliações das Vocalizações. As vocalizações foram avaliadas por dois observadores, a própria pesquisadora e um observador independente e foram reavaliadas as discordâncias até obter-se um índice de concordância de 90\% (Delgado \& Bevilacqua, 1999; Kazdin, 1982).

A avaliação das vocalizações dos participantes foi feita com base em Anastácio-Pessan et al. (2015), utilizando-se quatro categorias: correspondência total, correspondência parcial, emissão sem correspondência e omissões, assim definidas: (a) Correspondência total: vocalizações emitidas com correspondência ponto-a-ponto com o uso convencional. (b) Correspondência parcial: palavras vocalizadas com trocas fonêmicas ou quando o participante emitia todos os fonemas, contudo alterava o som de algum dos fonemas em nasalação ou tonicidade. (c) Sem correspondência: vocalizações de outra palavra que não possuía nenhum fonema em comum com a convencionada. Exemplo: diante da figura "lua", o participante vocalizou /sol/. (d) Omissões: quando o participante não emitia nenhuma vocalização após a apresentação da figura ou palavra escrita.

\section{Critério para Passagem de Fase}

Estabeleceu-se o critério de $90 \%$ de acertos em uma sessão para que os participantes pudessem passar de uma fase de ensino para outra. Não foi estabelecido critério nas fases de teste.

\section{Resultados}

Os resultados são apresentados por fase. Os resultados das Fases 1 e 7, pré e pós-teste, podem ser vistos na Figura 2, apresentados de acordo com o número de sílabas das palavras. 
No pré-teste de vocalizações a participante Ana, com maior tempo de privação auditiva, apresentou mais omissões na tarefa de nomeação de figuras, com desempenho mais preciso diante de figuras com nomes dissílabos e desempenho menos preciso diante de figuras com nomes monossílabicos. As omissões apresentadas por Ana no pré-teste foram diante das figuras de tartaruga, balanço, cruz e trem

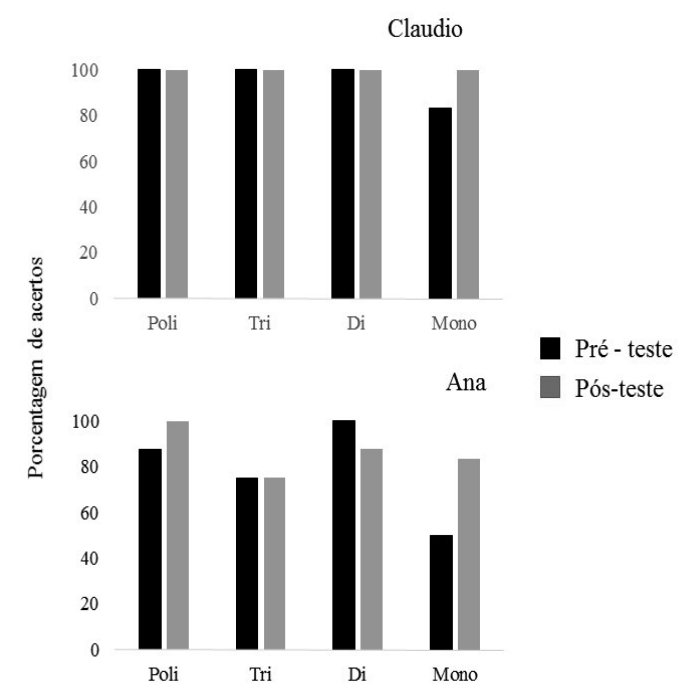

Figura 2. Porcentagem de acertos dos participantes na tarefa de nomeação de figuras no Pré-Teste, Fase 1, e no Pós-Teste, Fase 7, por número de sílabas das palavras.

No pós-teste, Ana apresentou duas omissões na tarefa de nomeação, diante das figuras de balanço e lixo, e uma vocalização sem corres- pondência diante da figura de flor, cuja vocalização foi /sol/.

Apresentou aumento na correspondência verbal em vocalizações diante de figuras com nomes polissilábicos e monossilábicos, tendo, especificamente, maior número de vocalizações com correspondência total diante de figuras com nomes polissilábicos, e mais erros em nomes monossilábicos. Na tarefa de leitura, seu desempenho foi categorizado como correspondência total em palavras com todas as extensões silábicas, assim como no pré-teste.

No pré-teste, Fase 1, o participante Claudio apresentou vocalizações avaliadas como correspondência total diante de todas as figuras, exceto uma omissão diante de uma figura com nome monossilábico. No pós-teste, Fase 7, Claudio apresentou todas as vocalizações avaliadas como correspondência total. Na tarefa de leitura, seu desempenho manteve-se idêntico ao pré-teste.

Na Fase 2, pré-treino, os participantes apresentaram desempenho superior ao critério estabelecido, apresentando, em uma sessão, os repertórios pré-requisitos para as fases seguintes.

Nas Fases 3 e 4, todos os participantes aprenderam as relações auditivo-visuais entre palavras ditadas e figuras com os estímulos não-convencionados. Na Fase 3, Cláudio e Ana necessitaram de 13 e 14 exposições, respectivamente, ao bloco de relações entre sons e figuras não-convencionadas para alcançar o critério, respectivamente, como pode ser visto na Figura 3.
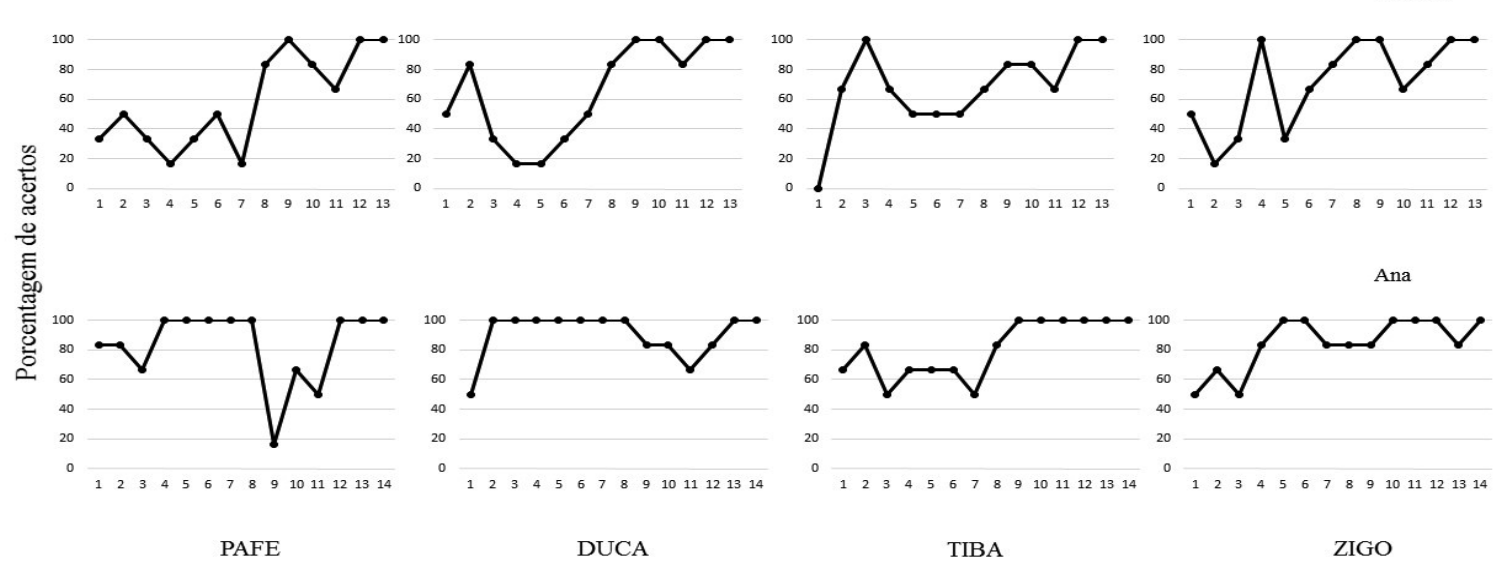

Figura 3. Desempenho dos participantes por relação, no treino das relações indefinidas entre modelo auditivo e figuras não convencionais na Fase 3. 
Os desempenhos dos participantes durante a Fase 3 foram acurados nas relações entre estímulos não-convencionados pelo procedimento de exclusão, mantiveram o responder de linha de base estável, e não responderam sob o efeito da novidade, isto é, responderam consistentemente à relação estabelecida na linha de base, podendose, portanto, descartar um possível efeito de controle pela novidade com a exposição ao Bloco 2 com estímulos não-convencionados, no entanto, no bloco 4 (de aprendizagem), necessitaram de mais exposições até alcance do critério.

Na Fase 4, treino das relações entre palavras ditadas e palavras escritas, os participantes apresentaram desempenho de $100 \%$ de acertos em uma única exposição ao treino.

No teste de formação de classe, Fase 5, os participantes evidenciaram a expansão das classes com a emergência de relações que não foram diretamente ensinadas. $\mathrm{O}$ participante Claudio apresentou desempenho de $91,6 \%$ de acertos nas tentativas de teste das relações entre figuras como estímulos-modelo e palavras escritas como estímulos-comparação (BC) e $100 \%$ de acertos nas tentativas de teste para relacionar palavras escritas e figuras (CB), enquanto que a participante Ana apresentou desempenho de $100 \%$ em todos os tipos de tentativas.

No teste de generalização, todos os participantes apresentaram desempenho geral de $100 \%$ de acertos em uma única sessão. Os participantes relataram que não perceberam os estímulos auditivos apresentados como voz masculina, apesar de sinalizarem ser uma voz diferente da apresentada durante o treino.

\section{Discussão}

Os objetivos de investigar se adultos usuários de implante coclear com tempos diferentes de privação auditiva (a) apresentariam desempenho acurado de discriminações condicionais auditivo-visuais com estímulos convencionados e não-convencionados; (b) se formariam as classes de estímulos consistentes com as relações aprendidas; (c) se a função dos estímulos auditivos se generalizariam para outra frequência auditiva; e (d) se estes repertórios afetariam o comportamento de falante, foram atingidos.

Os dados obtidos replicam os da literatura, demonstrando a eficácia do procedimento de pareamento ao modelo auditivo-visual, fading e exclusão para o ensino de novos repertórios com população com repertório verbal mínimo (Bagaiolo \& Micheletto, 2004).

Embora os participantes tivessem cinco meses (Cláudio) e nove anos (Ana) de privação auditiva, ambos apresentaram comportamento de falante com altos níveis de correspondência com a comunidade verbal nas tarefas de vocalização, ainda que com certa defasagem em relação ao padrão de fala normal. Os dados obtidos nos testes de vocalização corroboram os de Battaglini, Almeida-Verdu e Bevilacqua (2013) e Golfeto e de Souza (2015) sobre emissão de vocalizações com maior correspondência na tarefa de leitura de palavras do que na tarefa de nomeação de figuras. Battaglini et al. (2013) argumentam que a leitura de palavras é favorecida pela correspondência gráfica da palavra ditada em que a sequência de grafemas corresponde à de sons, isto é, o controle visual especifica cada parte da vocalização a ser emitida.

Os participantes aprenderam as relações entre os estímulos auditivos e figuras de treino, formando classes de estímulos auditivos, figuras e palavras escritas correspondentes. Do ponto de vista de utilidade do IC, este resultado confirma estudos anteriores (Anastácio-Pessan et al., 2015; Golfeto \& de Souza, 2015) que mostraram que o implante de IC reconstitui a sensibilidade ao som, mas as discriminações auditivo-visuais e o responder consistentemente aos sons associados a figuras e palavras escritas precisa ser treinado, no caso de pacientes pré-linguais, e re-treinado nos pós-linguais, para atingir a competência de um falante-ouvinte capaz de se comunicar, ler, conversar com outras pessoas sob controle das relações entre os sons e os demais eventos.

Os dados obtidos concordam com os dados apresentados na literatura que mostram que tanto deficientes auditivos pré-linguais quanto póslinguais, usuários de IC, apresentam ganhos na correspondência com a comunidade verbal após treino ostensivo de repertório de ouvinte (Almei- 
da-Verdu et al., 2008; Anastácio-Pessan et al., 2015; Golfeto \& de Souza, 2015).

Ao relacionar o tempo de privação auditiva com o desempenho de discriminação auditiva, os dados obtidos neste estudo também concordam com os dados apresentados na literatura de que quanto maior o tempo de privação auditiva, maior a dificuldade de reabilitação auditiva, observado na diferença de alcance de critério dos participantes do estudo de Anastácio-Pessan et al. (2015), por exemplo, que necessitaram, em sua maioria, de uma exposição ao bloco, enquanto que os participantes deste estudo necessitaram de 13 e 14 exposições. Dados com maior número de implantados pós-linguais são, entretanto, necessários para estabelecer essa correlação.

Enquanto os estudos sobre a aquisição e funcionamento do comportamento simbólico na literatura geralmente contaram com a participação de deficientes auditivos pré-linguais, especialmente crianças, os participantes deste estudo foram adultos deficientes auditivos pós-linguais, uma população diferente, mas igualmente carente de atendimento. E mesmo com esta população, que se pressupõe ter o desempenho linguístico já desenvolvido, discute-se que os operantes verbais envolvidos nas habilidades de falante e ouvinte, pós-implante, parecem necessitar de condições específicas de aprendizagem (Almeida-Verdu et al., 2008; Wei, Cao, Jin, Chen, \& Zeng, 2007).

Considerando os resultados satisfatórios, parece ter sido relevante demonstrar que pessoas com deficiência auditiva pós-lingual, com implante coclear após diferentes tempos de privação da audição, uma vez expostos a um programa de ensino de habilidades de ouvinte e de falante, de discriminação de sons longos e curtos, podem aprender relações simbólicas novas entre estímulos auditivos e visuais.

Os resultados positivos nos testes de generalização de frequências auditivas indicam que o implante coclear é capaz de proporcionar ao seu usuário sensibilidade auditiva suficiente para discriminar palavras em tons de voz diferentes do usado no ensino. Este repertório é funcional na vida diária do ser humano, no trânsito, no trabalho, em casa, etc., pois no cotidiano, diferentes sons devem ser reconhecidos como funcionalmente iguais, como sons de buzinas, de telefone, etc. Uma mensagem deve ser reconhecida em diferentes frequências de voz, assim como espera-se que uma pessoa ouvinte seja capaz de atender a alguém chamando à porta, seja ouvindo baterem na porta, baterem palmas ou acionarem a campainha, e assim por diante. No entanto, os relatos dos participantes demonstraram fraca discriminação entre voz masculina e feminina. Este dado aponta para a necessidade de novos estudos para avaliar e treinar discriminações mais acuradas entre timbres e entonações.

Os autores também sugerem que estudos futuros utilizem ruído ambiental como estímulo discriminativo, para aproximar o ambiente experimental da realidade dos participantes, e a apresentação de estímulos onomatopeicos, que podem facilitar os primeiros passos para a reabilitação desta população com audição recente.

Uma dificuldade dos estudos com pessoas com IC realizados da perspectiva do paradigma de equivalência de estímulos é sua longa duração, com grande número de fases e de tentativas por fase (Almeida-Verdu et al., 2008; Anastácio-Pessan et al., 2015; Golfeto \& de Souza, 2015). Levando em consideração essa dificuldade, neste estudo buscou-se adotar um procedimento com pequeno número de fases e de tentativas em cada fase, procurando-se evitar o cansaço e a eventual desistência ao longo do estudo.

Uma limitação deste e outros estudos envolvendo usuários de IC relaciona-se com o procedimento empregado de inserir uma sequência de contingências de treino das habilidades de ouvinte e testar o efeito do treino de ouvinte sobre o de falante somente após o treino. Este procedimento entra em conflito com o desenvolvimento da linguagem em ambiente natural, que nas relações com os adultos, as crianças, com desenvolvimento típico, aprendem comportamentos de falante e ouvinte de modo alternados. Apresentar um estímulo vocal, o nome de um objeto, por exemplo, na presença do objeto e da criança, pode ser condição favorável para que a criança aprenda, via reforçamento social, a realizar comportamentos convencionais em relação ao objeto. Horne e Lowe (1996) 
defendem que o ecoico seria um elo importante no estabelecimento de comportamento de ouvinte e falante. Assim, quando diante de um estímulo auditivo a criança responde ecoando, o cuidador reforça esse desempenho (Skinner, 1957). No entanto, após a criança já ter adquirido o comportamento de ouvinte, o estímulo auditivo, além do ecoico, também ocasionará o comportamento de ouvinte da criança. Neste ponto a criança se torna uma falante-ouvinte em relação ao seu próprio estímulo verbal. Outro elemento pertencente ao desenvolvimento do comportamento de falante, é o operante verbal tato, no qual uma resposta, por exemplo de nomear, é evocada por um evento em particular (Horne \& Lowe, 1996).

No estabelecimento de habilidades verbais, a forma mais provável do tato ocorrer é através do ecoico e comportamento de ouvinte previamente estabelecidos (Horne \& Lowe, 1996).

Estudos futuros podem apresentar procedimentos em que as habilidades de ouvinte e falante sejam apresentadas de modo intercalado em uma mesma sessão. $\mathrm{O}$ procedimento de Instrução por Exemplares Múltiplos (MEI) tem sido promissor em desenvolver repertórios verbais de falante e ouvinte em crianças com desenvolvimento atípico (Greer \& Ross, 2008).

O presente estudo buscou colaborar na geração de tecnologia comportamental aplicável em ambientes terapêuticos e educacionais, favorecendo a reabilitação auditiva com um programa de ensino com eficácia demonstrada na literatura. Adicionalmente, contribuiu com o conhecimento de condições em que deficientes auditivos pós-linguais implantados podem adquirir competência simbólica através do planejamento de procedimentos de ensino efetivos em produzir a expansão do repertório verbal.

\section{Referências}

Almeida-Verdu, A. C., Huziwara, E. M., de Souza, D. G., de Rose, J. C., Bevilacqua, M. C., Lopes, J., \& McIlvane, W. J. (2008). Relational learning in children with deafness and cochlear implants. Journal of the Experimental Analysis of Behavior, 89, 407-424. doi:10.1901/jeab.200889-407
Almeida-Verdu, A. C. M., de Souza, D. G., Bevilacqua, M. C., \& Souza, F. C. (2009). Imitação vocal e nomeação de figuras em deficientes auditivos usuários de implante coclear: Estudo exploratório. Revista Brasileira de Análise do Comportamento, 5, 63-78.

Anastácio-Pessan, F. L., Almeida-Verdu, A. C. M., Bevilacqua, M. C., \& Souza, D. G. (2015). Usando o paradigma de equivalência para aumentar a correspondência na fala de crianças com implante coclear na nomeação de figuras e na leitura. Psicologia: Reflexão e Crítica, 28, 365-377. doi:10.1590/1678-7153.201528217

Bagaiolo, L. F., \& Micheletto, N. (2004). Fading e exclusão: Aquisição de discriminações condicionais e formação de classes de estímulos equivalentes. Temas em Psicologia, 12, 168-185. Recuperado em http://pepsic.bvsalud.org/scielo. php?script $=$ sci_arttext\&pid=S1413-389X20040 $00200008 \& \operatorname{lng}=\mathrm{pt} \& \mathrm{t} \operatorname{lng}=\mathrm{pt}$

Battaglini, M. P., Almeida-Verdu, A. C. M., \& Bevilacqua, M. C. (2013). Aprendizagem via exclusão e formação de classes de equivalência em crianças com deficiência auditiva e implante coclear. Acta Comportamentalia, 21, 20-35.

Bevilacqua, M. C. (1998). Implante coclear multicanal: Uma alternativa na habilitação de crianças surdas (Tese de Livre Docência, Universidade de São Paulo, Faculdade de Odontologia de Bauru, SP, Brasil).

Clark, G. M. (1996). Electrical stimulation of the auditory nerve: The coding of frequency, the perception of pitch and the development of cochlear implant speech-processing strategies for profoundly deaf people. Clinical and Experimental Pharmacology and Physiology, 23, 766-776.

Da Silva, W. R., de Souza, D. G., de Rose, J. C., Lopes, J., Jr., Bevilacqua, M. C., \& McIlvane, W. J. (2006). Relational learning in deaf children with cochlear implants. Experimental Analysis of $\mathrm{Hu}$ man Behavior Bulletin, 24, 1-8.

Delgado, E. M. C., \& Bevilacqua, M. C. (1999). Lista de palavras como procedimento de avaliação da percepção dos sons da fala para crianças deficientes auditivas. Pró-fono, 11, 59-64.

Dixon, L. S. (1977). The nature of control by spoken words over visual stimulus selection. Journal of the Experimental Analysis of Behavior, 27, 433442. doi:10.1901/jeab.1977.27-433 
Golfeto, R. M., \& de Souza, D. G. (2015). Sentence production after listener and echoic training by prelingual deaf children with cochlear implants. Journal of Applied Behavior Analysis, 48, 363-375. doi:10.1002/jaba.197

Greer, R. D., \& Ross, D. E. (2008). Verbal behavior analysis: Inducing and expanding new verbal capabilities in children with language delays. Boston, MA: Pearson Education.

Horne, P. J., \& Lowe, C. F. (1996). On the origins of naming and other symbolic behavior. Journal of the Experimental Analysis of Behavior, 65, $185-$ 241.

Kazdin, A. E. (1982). Single-case research designs: Methods for clinical and applied settings. New York: Oxford University Press.

McIlvane, W. J., Munson, L. C., \& Stoddard, L. T. (1988). Some observations on control by spoken words in children's conditional discrimination and matching by exclusion. Journal of Experimental Child Psychology, 45, 472-495.

McIlvane, W. J., \& Stoddard, L. T. (1981). Acquisition of matching-to-sample performances in severe retardation: Learning by exclusion. Journal of Mental Deficiency Research, 25, 33-48.

Neves, A. J. D. (2014). Compreensão e produção oral de sentenças em crianças com deficiência auditiva pré-lingual usuárias de implante coclear (Dissertação de mestrado, Universidade Estadual Paulista Júlio de Mesquita Filho, Faculdade de Ciências, São Paulo, SP, Brasil). Recuperado em http://hdl.handle.net/11449/132489
Neves, A. J. D., Verdu, A. C. M. A., MortariMoret, A. L., \& Silva, L. T. D. N. (2015). As implicações do implante coclear para desenvolvimento das habilidades de linguagem: uma revisão da literatura. Revista CEFAC, 17, 16431656. doi:10.1590/1982-021620151755315

Skinner, B. F. (1957). Verbal behavior. Englewood Cliffs, NJ: Prentice Hall.

Souza, G. N., Jr. (2010). Projeto de software para aprendizagem utilizando discriminação simples simultânea. Trabalho de Conclusão de Curso de Bacharel em Sistema de Informação, Faculdade de Computação, Instituto de Ciências Exatas e Naturais, Universidade Federal do Pará, Belém, PA, Brasil.

Souza, F. C. de, Almeida-Verdu, A. C. M., \& Bevilacqua, M. C. (2013). Ecoico e nomeação de figuras em crianças com deficiência auditiva pré-lingual com implante coclear. Acta comportamentalia, 21, 325-339.

Wei, C., Cao, K., Jin, X., Chen, X., \& Zeng, F. G. (2007). Psychophysical performance and Mandarin tone recognition in noise by cochlear implant users. Ear and Hearing, 28(Suppl. 2), 62S-65S. doi:10.1097/AUD.0b013e318031512c

Recebido: 04/12/2015

$1^{a}$ revisão: $24 / 06 / 2016$

Aceite final: 26/07/2016 